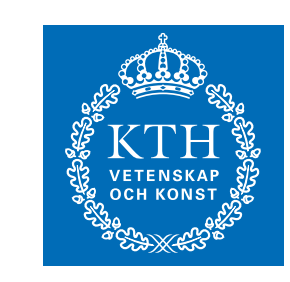

КTH Electrical Engineering

\title{
Lattice-Based Source-Channel Coding in Wireless Sensor Networks
}

(C) 2011 IEEE. Personal use of this material is permitted. However, permission to reprint/republish this material for advertising or promotional purposes or for creating new collective works for resale or redistribution to servers or lists, or to reuse any copyrighted component of this work in other works must be obtained from the IEEE.

JOHANNES KARLSSON AND MIKAEL SKOGLUND

Stockholm 2011

School of Electrical Engineering and the ACCESS Linnaeus Center, Royal Institute of Technology (KTH), SE-100 44 Stockholm, Sweden 


\title{
Lattice-Based Source-Channel Coding in Wireless Sensor Networks
}

\author{
Johannes Karlsson and Mikael Skoglund \\ School of Electrical Engineering and the ACCESS Linnaeus Center \\ Royal Institute of Technology (KTH), Stockholm, Sweden. \{johk, skoglund\}@ee.kth.se
}

\begin{abstract}
We consider the problem of gathering measurements in a wireless sensor network consisting of a large number of sensor nodes. A practical joint source-channel coding scheme is proposed and evaluated. The scheme uses lattices to extend a previously proposed scheme to higher dimensions. The key idea is to use conventional point-to-point communication for a subset of the sensor nodes and side-information aware transmission for the remaining sensor nodes. The selection of sensors is based on their instantaneous channel quality. It is shown that by expanding from one to eight dimensions, a gain of about $1 \mathrm{~dB}$ is achievable. The overall transmission delay of the scheme is still very low and it is therefore suitable to use in delay-sensitive applications.
\end{abstract}

\section{INTRODUCTION}

We consider the important task of communicating correlated sensor measurements to a fusion center over noisy channels. We will assume that the sensor measurements are real and thus take values from an infinite alphabet. This is a lossy distributed source-channel coding problem. Lossy source coding with side-information was first studied by Wyner and Ziv in [1], where they characterize the rate-distortion region when side information is available at the decoder. This problem has been extended to other scenarios of lossy distributed source coding, such as multi-terminal source coding [2], [3] and the Chief Executive Officer (CEO) problem [4]. In multi-terminal source coding in general, the objective is to estimate an entire field at the fusion center, where each sensor can measure only a part of the field. Whereas in the CEO problem, all sensors have noisy observations of the same source and the objective is to estimate this single underlying source at the fusion center. In the following we will consider the first of these problems, namely, to estimate an entire field.

The rate-distortion region of this problem is only known in a few special cases, such as the quadratic Gaussian twoencoder problem [5]. It is, in general, very difficult to find the rate-distortion region for larger networks. Nevertheless, there are numerous upper and lower bounds, see [2], [3], [5]-[7].

In this paper, we will assume that the communication channels are made orthogonal by either frequency- or timedivision multiple access techniques. It was recently proved [8] that the separation of source and channel coding, which is optimal in point-to-point communication, also is optimal in this scenario. However, the focus of our work is on delaysensitive applications, such as networked control systems.

This work was funded in part by VINNOVA and the Swedish Research Council.
In these applications the measurements are used in active decisions and there is a penalty involved in receiving delayed measurements; the system might even become unstable in the worst case. The information theoretic arguments for optimal source and channel coding and the separation principle, are all based on block lengths that asymptotically tend to infinity. It is clear that long block lengths, which would lead to significant decoding delays, does not fulfill our low-delay constraints. Therefore, we propose the use of a joint source-channel code, operating on short blocks of only a few samples, which gives virtually no transmission delay at all.

Uncoded linear transmission has been shown to outperform schemes based on separate source and channel codes if specific conditions are fulfilled [9]. Because of this, linear transmission may seem to be a good candidate of a joint source-channel code for the problem at hand, see [10]-[12] for examples where this is used. However, since the channels are orthogonal and the measurements are correlated (as in [10]-[12]), a linear transmission scheme will only use a subspace of the channel; in the limit of high correlation, the subspace will be onedimensional, which clearly is a waste of energy.

In [13], we considered a pair of sensors and proposed a design algorithm that jointly, in an iterative manner, optimized the operation at each sensor node as well as the decoder at the fusion center. This resulted in highly optimized systems that, in general, were nonlinear. The nonlinearities were such that all dimensions of the channel space were utilized. However, although straightforward in theory, the generalization to more than a couple of sensor nodes or vector measurements is infeasible due to high computational complexity. To reduce the complexity, we need some structure. A practical analog scheme that uses piecewise linear mappings was presented in [14]. The simple encoding and decoding structure made it possible to handle an arbitrary number of sensor nodes.

We generalize the ideas of piecewise linear mappings to higher dimensions by using modulo-lattice modulation (MLM). In addition, we also include fading channels in our model and present an idea for scheduling of the sensor nodes. Lattices have been proposed and analyzed as a tool to do structured distributed source coding in WSNs before, see for example [15]-[18]. However, most results are theoretical and asymptotic in some sense. The idea of MLM originates from nested lattice codes in digital systems [17], [18] and was mentioned in [15] as a means of performing analog communication with side information. The idea is analyzed in [16] in a point- 
to-point scenario, where it is shown that MLM asymptotically reaches the Wyner-Ziv bound as the lattice dimension goes to infinity. Another motivation for MLM in situations where side information is available at the decoder can be found in [19]. In this case a scalar relay mapping was numerically optimized and the resulting mapping is very similar to a sawtooth curve, which is the one-dimensional equivalence of MLM. Motivated by low-delay constraints, we will look at finite-dimensional systems and especially investigate the gains in a practical system when the dimensions are increased. In what follows, we propose a practical and scalable lattice-based strategy for collecting sensor measurements in a WSN consisting of a few to hundreds of sensors.

\section{Problem Formulation}

We study a complete WSN with $K$ sensor nodes as illustrated in Figure 1. Each sensor measures an $N$-dimensional quantity

$$
\boldsymbol{Y}_{i}=\boldsymbol{X}+\boldsymbol{W}_{i} \in \mathbb{R}^{N} \quad i=1, \ldots, K,
$$

where $\boldsymbol{X}$ and $\boldsymbol{W}_{i}$ are independent Gaussian random variables with covariance matrices $\sigma_{X}^{2} \boldsymbol{I}$ and $\sigma_{W_{i}}^{2} \boldsymbol{I}$, respectively. Hence, the samples are spatially correlated but temporally uncorrelated. We would like to emphasize that we are considering the multi-terminal source coding problem of estimating a random field, which is modeled by $\boldsymbol{Y}_{i}, i=1, \ldots, K$. That is, the objective is to estimate each sensor's observation at the fusion center and we are not interested in $\boldsymbol{X}$, except to the extent it can be helpful in estimating $\boldsymbol{Y}_{i}, i=1, \ldots, K$. Each sensor encodes its measurement independently in blocks of length $N$ according to the function

$$
\boldsymbol{s}_{i}\left(\boldsymbol{y}_{i}\right): \mathbb{R}^{N} \mapsto \mathbb{R}^{N} \quad i=1, \ldots, K,
$$

which is then transmitted to the fusion center over a Rayleigh block fading channel with additive white Gaussian noise (AWGN). The received value from each sensor is denoted by $\boldsymbol{R}_{i}$ and can be expressed as

$$
\boldsymbol{R}_{i}=H_{i} \boldsymbol{s}_{i}\left(\boldsymbol{Y}_{i}\right)+\boldsymbol{V}_{i} \quad i=1, \ldots, K,
$$

where $H_{i}$ is the real-valued Rayleigh fading coefficient with $E\left[\left|H_{i}\right|^{2}\right]=1$ and $\boldsymbol{V}_{i}$ is Gaussian noise with covariance matrix $\sigma_{V}^{2} \boldsymbol{I}$. At this point, we assume for simplicity that perfect channel-state information (CSI) is globally available. The fusion center estimates each $\boldsymbol{Y}_{i}$ from all received values as,

$$
\hat{\boldsymbol{Y}}_{i}=\boldsymbol{g}_{i}\left(\boldsymbol{R}_{1}, \boldsymbol{R}_{2}, \ldots, \boldsymbol{R}_{K}\right) \quad i=1, \ldots, K .
$$

To have a low-delay system we want the block length $N$ to be small. Typically, this will affect the performance and there is a tradeoff between delay and performance. As performance measure we use the mean squared error (MSE) per sample, defined as

$$
\mathrm{MSE} \triangleq \frac{1}{K N} \sum_{i=1}^{K} E\left[\left\|\boldsymbol{Y}_{i}-\hat{\boldsymbol{Y}}_{i}\right\|^{2}\right] .
$$

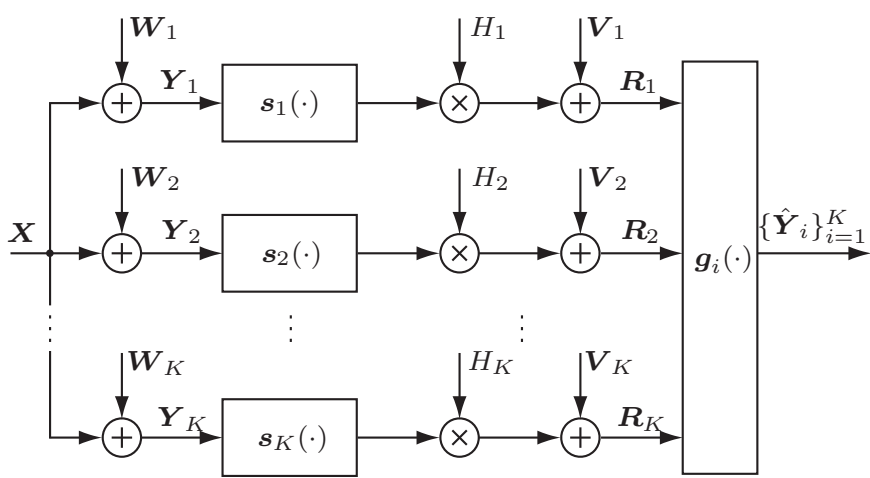

Fig. 1. Overview of the WSN. $K$ sensor nodes that should communicate their measurements to a fusion center.

The objective is to minimize the MSE by a proper choice of encoding and decoding functions $\left\{\boldsymbol{s}_{i}, \boldsymbol{g}_{i}\right\}_{i=1}^{K}$, where $\boldsymbol{s}_{i}$ should satisfy an average power constraint

$$
\frac{1}{N} E\left[\left\|\boldsymbol{s}_{i}\left(\boldsymbol{y}_{i}\right)\right\|^{2}\right] \leq P \quad i=1, \ldots, K .
$$

\section{PROPOSED SCHEME}

Due to fading, the signal-to-noise ratio (SNR) of the different channels to the fusion center will be different. Some sensors will have a high SNR, which could correspond to the sensor being close to the fusion center, line-of-sight transmission or constructive multipath propagation; whereas others have a low SNR, which could correspond to the sensors being further away, something blocking their transmission or destructive multipath propagation. Distributed source coding is mainly a way to reduce transmission rate or equivalently, in analog transmission as studied in this paper, a way to mitigate the effects of the channel noise. Because of this and since we are interested in each sensor's measurement, there is little gain in using distributed source coding for the sensors that already have a high SNR. We therefore propose the following scheduling and transmission strategy:

1) Transmit the measurements from the $M$ sensors having highest SNR with conventional point-to-point sourcechannel coding techniques. In this scenario we use linear transmission.

2) Estimate $\boldsymbol{X}$ and $\boldsymbol{Y}_{i}, i=1, \ldots, M$, from these transmissions.

3) Transmit the measurements from the remaining sensors using MLM, assuming that the estimate of $\boldsymbol{X}$ is available as side information at the receiver.

\section{A. Phase 1: Linear Transmission}

Without loss of generality, let the sensors be in such way that linear transmission is used for the $M$ first sensor nodes, that is,

$$
\boldsymbol{s}_{i}\left(\boldsymbol{y}_{i}\right)=\sqrt{\frac{P}{\sigma_{X}^{2}+\sigma_{W_{i}}^{2}}} \boldsymbol{y}_{i} \quad i=1, \ldots, M .
$$




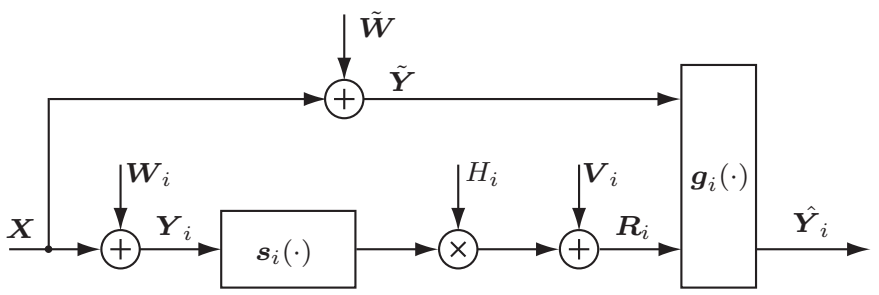

Fig. 2. Transmission from one isolated sensor node using MLM with side information at the receiver.

The optimal receiver given the $M$ linear transmissions is obtained by solving the Wiener-Hopf equations [20]. Let the elements of a vector $\boldsymbol{A}_{i}$ be denoted by $A_{i j}, j=1, \ldots, N$ (e.g., $\boldsymbol{R}_{i}=\left[R_{i 1}, R_{i 2}, \ldots, R_{i N}\right]^{T}$ ). Each component of $Y_{i}$ can now be estimated by

$$
\hat{Y}_{i j}=\boldsymbol{t}^{(i)^{T}} \boldsymbol{R}^{(j)} \quad i=1, \ldots, M, j=1, \ldots, N,
$$

where

$$
\begin{aligned}
\boldsymbol{R}^{(j)} & =\left[R_{1 j}, R_{2 j}, \ldots, R_{K j}\right] \quad j=1, \ldots, N \\
\boldsymbol{t}^{(i)} & =\left(E\left[\boldsymbol{R}^{(j)} \boldsymbol{R}^{(j)^{T}}\right]\right)^{-1} E\left[Y_{i j} \boldsymbol{R}^{(j)}\right] \quad i=1, \ldots, M .
\end{aligned}
$$

In a similar manner, the optimal estimate of $\boldsymbol{X}$ is given by

$$
\hat{X}_{j}=\boldsymbol{t}^{(0)^{T}} \boldsymbol{R}^{(j)} \quad j=1, \ldots, N,
$$

where

$$
\boldsymbol{t}^{(0)}=\left(E\left[\boldsymbol{R}^{(j)} \boldsymbol{R}^{(j)^{T}}\right]\right)^{-1} E\left[X_{j} \boldsymbol{R}^{(j)}\right] .
$$

\section{B. Phase 2: Modulo-Lattice Modulation}

We need some basic terminology for lattices before we can proceed and look at the modulo-lattice modulation. An $N$ dimensional lattice $\Lambda$ is defined by the generator matrix $G \in$ $\mathbb{R}^{N \times N}$. A point $\boldsymbol{l} \in \mathbb{R}^{N}$ belongs to the lattice if and only if it can be written as $\boldsymbol{l}=G \boldsymbol{i}$, where $\boldsymbol{i} \in \mathbb{Z}^{N}$ and $\mathbb{Z}=$ $\{0, \pm 1, \pm 2, \ldots\}$. We define the nearest neighbor quantizer of a lattice $\Lambda$ by

$$
Q_{\Lambda_{\Delta}}(\boldsymbol{x}) \triangleq \arg \min _{\boldsymbol{l} \in \Lambda_{\Delta}}\|\boldsymbol{x}-\boldsymbol{l}\|
$$

where $\Delta$ is used to explicitly specify the minimum distance between lattice points (which is the same as two times the packing radius of the lattice). For a particular lattice $\Lambda$, any $\Delta$ can be achieved by a proper scaling of $G$. For the lattices considered in this paper, there exist highly efficient algorithms that perform the operation in (13) without the need of an exhaustive search. The modulo-lattice operation that will be used in the rest of the paper is defined by

$$
\boldsymbol{x} \bmod \Lambda_{\Delta} \triangleq \boldsymbol{x}-Q_{\Lambda_{\Delta}}(\boldsymbol{x}) .
$$

A comprehensive study of lattices and lattice quantization can be found in [21]. See also [16], where the MLM scheme is presented and analyzed in detail.

We will now present how we will use MLM in our scenario. Given the $M$ linear transmissions, we will isolate each of the remaining $K-M$ sensors and treat them individually as shown in Figure 2. The motivation for the individual treatment of the remaining sensors is the fact that $\boldsymbol{Y}_{i}$ and $\boldsymbol{Y}_{j}$ are conditionally independent given $\boldsymbol{X}$ for all $j \neq i$. The side-information from the linear transmissions is modeled by $\tilde{\boldsymbol{Y}}=\boldsymbol{X}+\tilde{\boldsymbol{W}}$, where $\tilde{\boldsymbol{W}}$ is Gaussian noise with covariance matrix $\sigma_{\tilde{W}}^{2} \boldsymbol{I}^{1}$. We use the correlation coefficients $\rho_{X Y}$ and $\rho_{X \tilde{Y}}$ to quantify the quality of the side-information,

$$
\rho_{X Y} \triangleq \frac{E\left[X_{j} Y_{i j}\right]}{\sigma_{X} \sigma_{Y_{i}}}, \rho_{X \tilde{Y}} \triangleq \frac{E\left[X_{j} \tilde{Y}_{j}\right]}{\sigma_{X} \sigma_{\tilde{Y}}} .
$$

Since side-information is available at the fusion center, we can use the following nonreversible modulo-operation at the source nodes

$$
\boldsymbol{s}_{i}\left(\boldsymbol{y}_{i}\right)=\beta_{i}\left(\alpha_{i} \boldsymbol{y}_{i} \bmod \Lambda_{\Delta_{i}}\right) \quad i=M+1, \ldots, K .
$$

There are three scaling parameters involved $-\alpha_{i}, \beta_{i}$, and $\Delta_{i}$. $\alpha_{i}$ is chosen such that the variance of each component of $\alpha_{i} \boldsymbol{Y}_{i}$ equals unity. In a similar way, $\beta_{i}$ is used to make sure that the power constraint in (6) is fulfilled. The parameter $\Delta_{i}$ specifies the minimum distance of lattice points and determines the amount of information that is removed prior to transmission. If $\Delta_{i}$ is large no information is removed and the sensor operates in a linear mode. On the other hand, if $\Delta_{i}$ is small, more information is removed and the fusion center would have to rely on the side-information to estimate the removed part and be able to decode.

The decoder is divided into three steps. First, the output of the sensor node, $\boldsymbol{s}_{i}\left(\boldsymbol{y}_{i}\right)$, is estimated from $\boldsymbol{R}_{i}$ by using the linear minimum MSE (MMSE) estimator,

$$
\hat{\boldsymbol{s}}_{i \mid R}=\frac{P h_{i}}{P h_{i}^{2}+\sigma_{V_{i}}^{2}} \boldsymbol{R}_{i} \quad i=M+1, \ldots, K .
$$

Next, the part that is removed during the modulo operation is estimated from $\hat{\boldsymbol{X}}$ and $\hat{\boldsymbol{s}}_{i \mid R}$,

$$
\hat{Q}_{\Lambda_{i}}=Q_{\Lambda_{\Delta_{i}}}\left(\alpha_{i} \hat{\boldsymbol{X}}-\frac{\hat{\boldsymbol{s}}_{i \mid R}}{\beta_{i}}\right) \quad i=M+1, \ldots, K .
$$

Finally, $\boldsymbol{Y}_{i}$ is estimated as

$$
\hat{\boldsymbol{Y}}_{i}=\frac{1}{\alpha_{i}}\left({\hat{Q_{\Lambda}}}_{\Lambda_{i}}+\frac{\hat{\boldsymbol{s}}_{i \mid R}}{\beta_{i}}\right) \quad i=M+1, \ldots, K .
$$

Provided that the side-information is sufficiently good such that $\hat{Q_{\Lambda_{i}}}=Q_{\Lambda_{\Delta_{i}}}\left(\alpha_{i} \boldsymbol{Y}_{i}\right)$ with high probability, decreasing $\Delta_{i}$ would allow the sensor to increase $\beta_{i}$ without violating the power constraint. This can be seen as a virtual power gain and decreases the MSE. However, if $\Delta_{i}$ is decreased below some threshold such that decoding errors become dominant in (18), the MSE will increase. The optimal $\Delta_{i}$ will depend both on $\rho_{X Y}$ and $\rho_{X \tilde{Y}}$ as well as the instantaneous SNR, $P h_{i}^{2} / \sigma_{V_{i}}^{2}$. We have performed a numerical grid search to find the optimal $\Delta_{i}$ for a large range of these parameters and the lattices $E_{8}$, $D_{4}, Z_{1}$, where the subindices in this case denote the lattice dimension. The resulting performance for $\rho_{X Y}=\rho_{X \tilde{Y}}=0.99$

\footnotetext{
${ }^{1} \tilde{\boldsymbol{Y}}$ can be obtained by a proper scaling of $\hat{\boldsymbol{X}}$ in (11).
} 


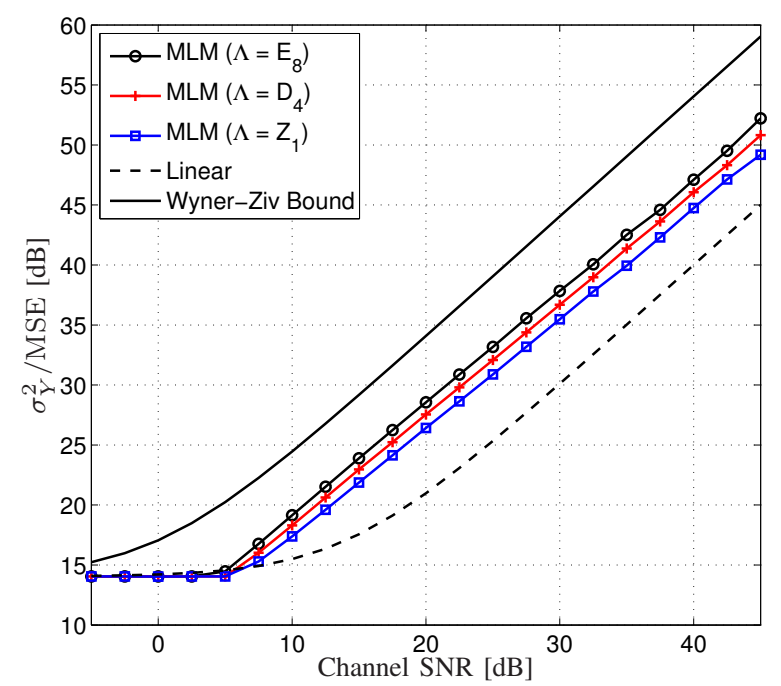

Fig. 3. Numerical results of single-link MLM using the lattices $E_{8}, D_{4}$ and $Z_{1}$. Linear transmission and the Wyner-Ziv bound are shown for comparison. $\rho_{X Y}=\rho_{X \tilde{Y}}=0.99$

can be seen in Figure 3, where we also compare the results to linear transmission and the Wyner-Ziv bound. The lattices we use $-Z_{1}, D_{4}$, and $E_{8}$ - are well known for their excellent properties. All of them are the densest lattice packings in their respective dimension and it is "a reasonable guess" [21] that they are also the best uniform quantizers among lattices. See [21] for details about their definitions and properties.

By looking at Figure 3 we can see that linear transmission is slightly better than MLM for SNR points less than 7-8 dB. Because of this we make a small modification to the selection of sensors that use linear transmission and include also those sensors with low SNR, where linear transmission is superior. As the SNR increases we see that the curves are more or less parallel. For the specified correlation, MLM with the lattice $E_{8}$ is $7-8 \mathrm{~dB}$ better than linear transmission. The gain of increasing the dimensions from 1 to 4 and from 4 to 8 is roughly $1 \mathrm{~dB}$. The gap to the Wyner-Ziv bound is $6-7 \mathrm{~dB}$, however, it is worth to stress that this bound assumes infinite dimensions in both the source and channel coding. In [16] it is in fact shown that MLM can reach the Wyner-Ziv bound as the lattice dimension goes to infinity.

\section{NUMERICAL RESULTS}

To evaluate the proposed method we will consider two scenarios - a WSN with 20 sensors and a WSN with 100 sensors. For each of these scenarios we let $\sigma_{W_{i}}^{2}=\sigma_{W}^{2}$ and simulate the system for two different correlation coefficients, namely $\rho_{X Y}=0.99$ and $\rho_{X Y}=0.995$. For the Rayleigh fading, we have assumed that the channel coefficients stay constant for 100 channel uses. The parameter $M$, which determines the number of sensors using linear transmission, has been optimized numerically for each SNR point. The results of the simulations for the two scenarios are presented in

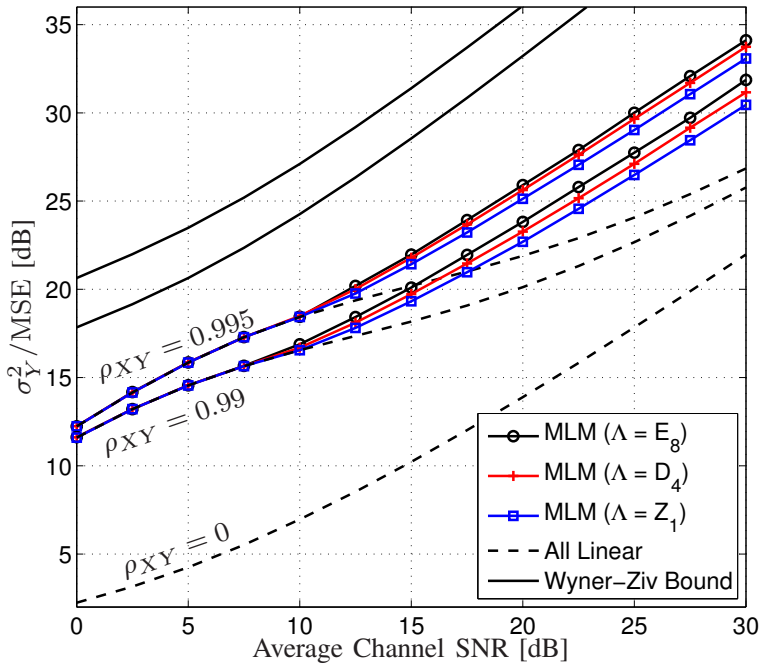

Fig. 4. WSN consisting of 20 sensor nodes. MLM in comparison to Linear transmission for $\rho_{X Y}=0.99$ and $\rho_{X Y}=0.995$.

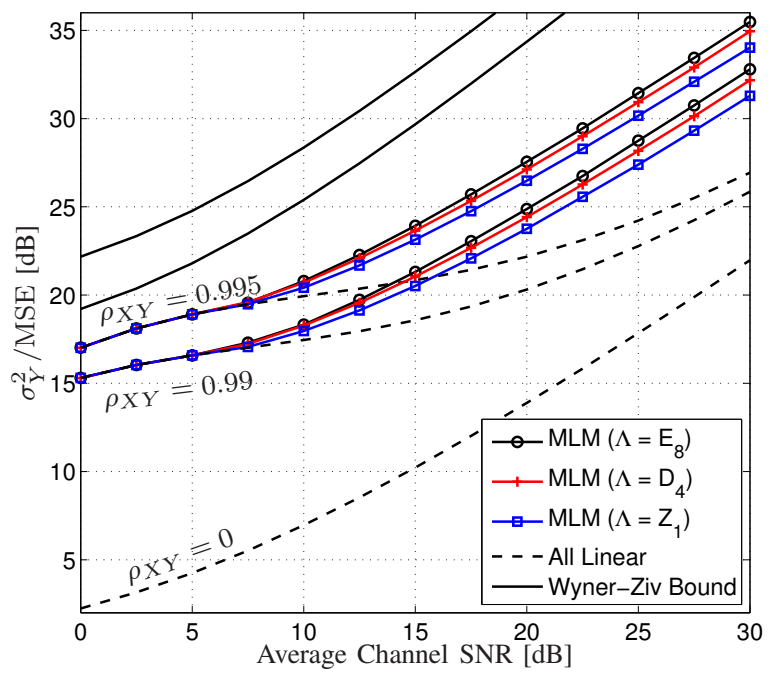

Fig. 5. WSN consisting of 100 sensor nodes. MLM in comparison to Linear transmission for $\rho_{X Y}=0.99$ and $\rho_{X Y}=0.995$.

Figure 4 and Figure 5. It is evident that our proposed method clearly outperforms transmission using linear modulation. As soon as the SNR exceeds a certain threshold, the gains are significant. This is despite the fact that the optimal receiver is used for the linear scheme whereas for the MLM scheme we have resorted to a suboptimal ad-hoc receiver. The limitation of the linear system is on the transmitting side. In the limit of a correlation coefficient $\rho_{X Y}=1$, for each sample, linear transmission only uses a one-dimensional subspace of the $K$-dimensional channel space. This is the explanation why the linear system is not able to take advantage of the increased correlation as the SNR increases; the curves for 


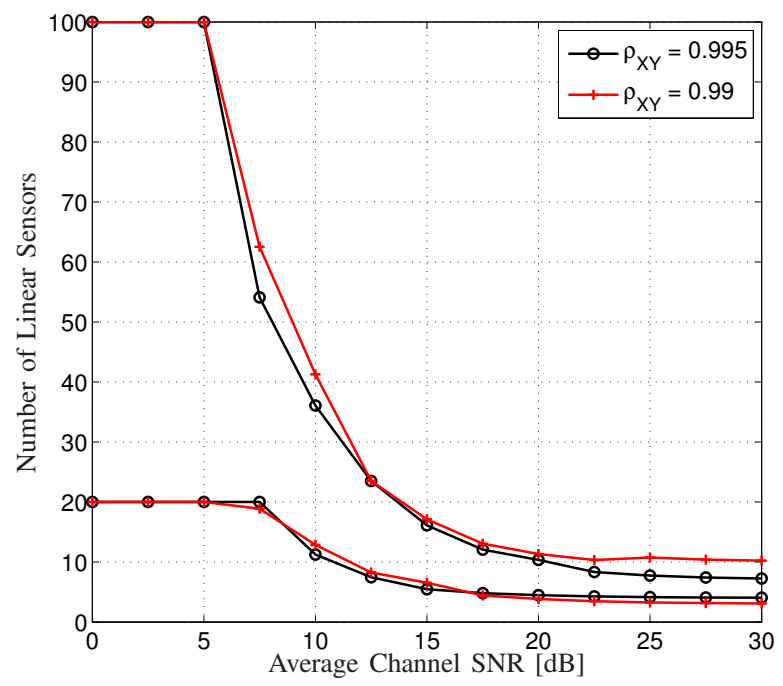

Fig. 6. Number of sensors that use linear transmission as a function of average channel SNR for MLM with $E_{8} \cdot \rho_{X Y}=0.99$ and $\rho_{X Y}=0.995$.

$\rho_{X Y}=0.99$ and $\rho_{X Y}=0.995$ asymptotically converge to the curve $\rho_{X Y}=0$. This is in contrast to the proposed system with MLM, where the advantage of a higher correlation is maintained as the SNR is increased. This is achieved by a decrease of $\Delta$ which makes sure that all dimensions of the channel space are used.

The Wyner-Ziv bound is obtained from Theorem 3 in [5], where the minimum MSE given a sum-rate constraint is stated. The bound is used by taking the sum of each link's ergodic channel capacity as the sum rate. In general, this bound is too optimistic and is not achievable with short block lengths since it relies on infinite block lengths in several senses. For example, there is no outage since we use the ergodic channel capacity, the effects of the channel noise is cancelled out by an optimal channel code, and the correlation behaves exactly as the correlation coefficient predicts.

Another observation is that the threshold where MLM performs better than linear transmission is shifted towards a lower SNR for the scenario with 100 sensors. This is explained by looking at Figure 6, where the number of linear sensors are plotted as a function of the SNR. At an SNR of $7.5 \mathrm{~dB}$ all sensors use linear transmission in the WSN with 20 sensors, while around 40 sensors use MLM in the WSN consisting of 100 sensor nodes. To put it in another way, as the number of sensor nodes increases, a smaller amount of sensors are needed to provide sufficiently good side information which is necessary for MLM to work.

\section{Conclusions}

We have proposed a joint source-channel coding scheme for WSNs based on MLM. The scheme is practical and has a low complexity that makes it suitable for WSNs consisting of a large number of sensors nodes. The use of very short block lengths is favorable in delay-sensitive applications such as control systems. We have investigated the gains of increasing the dimensions and shown that an 8-dimensional system performs about $1 \mathrm{~dB}$ better than a scalar system; linear transmission is clearly outperformed in all cases. The assumption of globally available CSI can easily be relaxed to a more realistic assumption of local CSI. The fusion center could then broadcast a threshold that determines if MLM or linear transmission should be used.

\section{REFERENCES}

[1] A. D. Wyner and J. Ziv, "The rate-distortion function for source coding with side information at the decoder," IEEE Trans. on Information Theory, vol. 22, no. 1, pp. 1-10, January 1976.

[2] T. Berger, "Multiterminal source coding," in The information theory approach to communications, G. Longo, Ed. Springer-Verlag, 1977.

[3] S.Y. Tung, "Multiterminal source coding," Ph.D. dissertation, Cornell University, Ithaca, NY, 1978.

[4] T. Berger, Z. Zhang, and H. Viswanathan, "The CEO problem [multiterminal source coding]," IEEE Trans. on Information Theory, vol. 42, no. 3, pp. 887-902, May 1996.

[5] A. B. Wagner, S. Tavildar, and P. Viswanath, "Rate region of the quadratic Gaussian two-encoder source-coding problem," IEEE Trans. on Information Theory, vol. 54, no. 5, pp. 1938-1961, May 2009.

[6] A. B. Wagner and V. Anantharam, "An improved outer bound for multiterminal source coding," IEEE Trans. on Information Theory, vol. 54, no. 5, pp. 1919-1937, May 2008.

[7] Y. Oohama, "Distributed source coding of correlated Gaussian observations," in Proceedings IEEE Int. Symp. Information Theory and its Applications, December 2008.

[8] J. J. Xiao and Z. Q. Luo, "Multiterminal source-channel communication over an orthogonal multiple-access channel," IEEE Trans. on Information Theory, vol. 53, no. 9, pp. 3255-3264, September 2007.

[9] M. Gastpar, B. Rimoldi, and M. Vetterli, "To code, or not to code: lossy source-channel communication revisited," IEEE Trans. on Information Theory, vol. 49, no. 5, pp. 1147-1158, May 2003.

[10] S. Cui, J. Xiao, A. Goldsmith, Z. Luo, and H. V. Poor, "Estimation diversity and energy efficiency in distributed sensing," IEEE Trans. on Signal Processing, vol. 55, no. 9, pp. 4683-4695, 2007.

[11] I. Bahceci and A. K. Khandani, "Linear estimation of correlated data in wireless sensor networks with optimum power allocation and analog modulation," IEEE Trans. on Communications, vol. 56, no. 7, pp. 11461156, 2008.

[12] J. Fang and H. Li, "Power constrained distributed estimation with correlated sensor data," IEEE Trans. on Signal Processing, vol. 57, no. 8, pp. 3292-3297, 2009.

[13] N. Wernersson, J. Karlsson, and M. Skoglund, "Distributed quantization over noisy channels," IEEE Trans. on Communications, vol. 57, no. 6 , pp. 1693-1700, June 2009.

[14] N. Wernersson and M. Skoglund, "Nonlinear coding and estimation for correlated data in wireless sensor networks," IEEE Trans. on Communications, vol. 57, no. 10, pp. 2932-2939, October 2009.

[15] Z. Reznic, M. Feder, and R. Zamir, "Distortions bounds for broadcasting with bandwidth expansion," IEEE Trans. on Information Theory, vol. 52 no. 8, pp. 3778-3788, August 2006.

[16] Y. Kochman and R. Zamir, "Joint Wyner-Ziv/dirty-paper coding by modulo-lattice modulation," IEEE Trans. on Information Theory, vol. 55, no. 11 , pp. 4878-4889, November 2009.

[17] R. Zamir and S. Shamai (Shitz) and U. Erez, "Nested linear/lattice codes for structured multiterminal binning," IEEE Trans. on Information Theory, vol. 48, no. 6, June 2002.

[18] S. D. Servetto, "Lattice quantization with side information: codes, asymptotics and applications in sensor networks," IEEE Trans. on Information Theory, vol. 53, pp. 714-731, feb 2007.

[19] J. Karlsson and M. Skoglund, "Optimized low-delay source-channelrelay mappings," IEEE Trans. on Communications, no. 5, pp. 13971404, May 2010.

[20] S. M. Kay, Fundamentals of statistical signal processing: estimation theory. Upper Saddle River, NJ, USA: Prentice-Hall, Inc., 1993.

[21] J. H. Conway and N. J. A. Sloane, Sphere packings, lattices and groups. New York, USA: Springer-Verlag, 1988. 